\title{
Human Conscious Experience is Four- Dimensional and has a Neural Correlate Modeled by Einstein's Special Theory of Relativity
}

\begin{abstract}
Richard Allen Sieb
ABSTRACT

In humans, knowing the world occurs through spatial-temporal experiences and interpretations. Conscious experience is the direct observation of conscious events. It makes up the content of consciousness. Conscious experience is organized in four dimensions. It is an orientation in space and time, an understanding of the position of the observer in space and time. A neural correlate for four-dimensional conscious experience has been found in the human brain which is modeled by Einstein's Special Theory of Relativity. Spacetime intervals are fundamentally involved in the organization of coherent conscious experiences. They account for why conscious experience appears to us the way it does. They also account for assessment of causality and past-future relationships, the integration of higher cognitive functions, and the implementation of goal-directed behaviors. Spacetime intervals in effect compose and direct our conscious life. The relativistic concept closes the explanatory gap and solves the hard problem of consciousness (how something subjective like conscious experience can arise in something physical like the brain). There is a place in physics for consciousness. We describe all physical phenomena through conscious experience, whether they be described at the quantum level or classical level. Since spacetime intervals direct the formation of all conscious experiences and all physical phenomena are described through conscious experience, the equation formulating spacetime intervals contains the information from which all observable phenomena may be deduced. It might therefore be considered expression of a theory of everything.
\end{abstract}

Key Words: conscious experience, time, space, four-dimensional, relativity, spacetime interval DOI Number: 10.14704/nq.2016.14.3.983 NeuroQuantology 2016; 4:630-644

\section{Introduction}

Conscious experience is defined as the direct observation of conscious events. It makes up the content of consciousness, ie., what is in consciousness (Sieb, 2004, 2013, 2015, 2016). Qualia are qualities or properties perceived or experienced by a person. They include such things as color, shape, texture, position, time, etc. A conscious event consists of a set of qualia. A ball (round, red, spongy, etc.), car, computer, hand, shoe, chair, wall, and any other object viewed in consciousness, may be considered a conscious event. Perception is the identification, organization, and interpretation of sensory information in order to represent and understand the environment (Schacter et al., 2011); an awareness (experience) of the elements of the

Corresponding author: Richard Sieb, Ph.D.

Address: 11624-135St., Edmonton, Alberta, Canada. T5M1K8

Phone: + 7802650095 , e-mail $₫$ siebr@shaw.ca

Relevant conflicts of interest/financial disclosures: The authors declare that the research was conducted in the absence of any commercial or financial relationships that could be construed as a potential conflict of interest.

Received: 25 February 2016; Revised: 14 July 2015; Accepted: 2 August 2015 
environment through physical sensation; physical sensation interpreted in the light of experience. Conscious experience is intimately tied to perception.

Some conscious events making up a conscious experience are observed simultaneously, but in different threedimensional spatial positions; other conscious events are observed at the same spatial positions, but at different times (just look around you). For example, when I examine my conscious experience of this room, many conscious events (door, window, light, light switch, walls, ceiling, floor, desk, chair, computer, monitor, keyboard, hands, mouse, gloves, etc.) are observed simultaneously, but in different threedimensional spatial positions. These events are separated only by space (since they are observed at different spatial positions), but not by time (since they are observed simultaneously). I observe other conscious events (my finger and the power button on the computer, my hand and the mouse, my hands and the gloves, a glass and a cup) at the same spatial positions (when I press the button, move the mouse, put the gloves on, or replace the glass with a cup), but at different times. These events are separated only by time (since they are observed at different times), but not by space (since they are observed at the same spatial positions). Notice that the conscious events separated by time also have cause-effect and pastfuture relations. Separation in time gives rise to causality and past-future relationships. This organization is typical and characteristic of conscious experience in general (all conscious experiences are organized in this manner) and indicates that conscious experience is organized in space and time. Since there are three dimensions of space (length, width, height) and one dimension of time, conscious experience can be said to have four dimensions. We observe (experience) the world in four dimensions.

In humans, knowing the world occurs through spatio-temporal experiences and interpretations (Maniadakis and Trahanias, 2011). Space and time play a critical role in the organization of conscious experiences. Space is the boundless three-dimensional extent in which "events" occur and have relative position and direction; time is a continuum in which "events" succeed one another from past through present to future. Emmanuel Kant prophetically concluded that space and time are not discovered by humans to be objective features of the world, but are part eISSN 1303-5150 of a systematic framework for the organization of our experiences (Lucas and Hodgson, 1985). This is made abundantly clear in what follows. Space is often conceived in three linear dimensions, but modern physicists usually consider it with time, as part of a boundless four-dimensional continuum called spacetime. Spacetime is any mathematical model that combines space and time into a single continuum (Petkov, 2010).

Einstein's special theory of relativity is the most successful model of spacetime. It depends on frames of reference (observational perspectives of space described using coordinate systems). Special relativity predicts a wide range of consequences (which have been experimentally verified). These include length contraction, time dilation, relativistic mass, mass-energy equivalence ( $E=m c^{2}$, where $c$ is the speed of light in a vacuum), a universal speed limit (c), and relativity of simultaneity (Disalle, 2009; Einstein, 2001; Feynman, 1998; Roberts and Schleif, 2007). Time dilates or lengthens (slows) and lengths contract (shorten) at higher speeds of a reference frame (observer) relative to another; this keeps the laws of physics, $c$, and spacetime intervals invariant (the same) in all inertial (constant velocity) frames of reference. In relativity, time cannot be separated from space, because the observed rate at which time passes depends on the relative velocity of the observer. Time and space are interwoven into a single continuum (the spacetime continuum). Since conscious experience is a spacetime continuum, Einstein's special theory of relativity is a viable model for conscious experience. A neural correlate for conscious experience has been found in the human brain which is modeled by Einstein's special theory of relativity (Sieb, 2016).

\section{Representation of Space}

In 1971, John O'Keefe discovered "place cells" in the hippocampus (O'Keefe and Dostrovsky, 1971; O'Keefe, 1976). Place cells fire at particular places in a spatially-structured environment. They fire so reliably one can tell where the subject is in an environment by observing which neurons are firing. The hippocampus acts as a neural representation of the layout of an environment. A place cell fires when the subject passes through a specific small region of space called the place field. Place fields are considered allocentric rather than egocentric because they are defined with respect to the outside world, rather than the body. By 
orientation based on the environment, place cells can work effectively as neural maps of the environment (Jeffery et al., 2003). The discovery of place cells led to a series of investigations that culminated in a book entitled The Hippocampus as a Cognitive Map, which argued that the hippocampal neural network instantiates cognitive maps for spatial memory function (O'Keefe and Nadel, 1978). This motivated hundreds of experimental studies aimed at clarifying the role of the hippocampus in spatial memory and spatial navigation (Moser and Moser, 1998; Maurer et al., 2005).

Considerable data indicate the hippocampus is critical to episodic memory in humans (Steinvorth et al., 2005; Rolls, 2013; Sieb, 2015, 2016). Episodic memory is memory of personal past experiences of a particular time and place with reference to the person as a participant-observer (Munoz-Lopez et al., 2010; Schacter et al., 2011; Sieb, 2015). Conscious recall is required to demonstrate episodic memory (Tulving, 1983; Ullman, 2004; Munez-Lopez et al., 2010); after all, episodic memory is memory of personal past conscious experiences. A fundamental feature of episodic memory is the spatial organization of events composing a unique experience (Serino and Riva, 2014). An allocentric representation of the environment is encoded in the hippocampus and stored as episodic memory and hippocampal place cells play a crucial role in the establishment and maintenance of this representation (Serino and Riva, 2014; Sieb, 2015, 2016). Place cells are part of a complex circuit that informs memory and awareness (Jeffery et al., 2003; Jeffery, 2007).

\section{Representation of Time}

Another fundamental feature of episodic memory is the temporal organization of serial events that compose a unique experience (MacDonald et al., 2011, abstract);

"The hippocampus is essential to encoding and remembering unique sequences of events as well as disambiguating sequences that share common events. Studies on humans have shown that the hippocampus is critical to remembering the flow of events in distinct experiences and, in doing so, bridges temporal gaps between noncontiguous events. There is a robust hippocampal representation of sequence memories, highlighted by time cells, that encode successive moments during empty temporal gaps between key events, while also encoding location and ongoing behavior."

\section{Representation of Spacetime}

The hippocampal representation of time is quite similar to its representation of space (MacDonald et al., 2011): a large proportion of neurons are engaged in both; time cells fire at discrete moments during "empty" periods in a temporallyorganized memory, much as place cells fire at discrete locations, devoid of specific stimuli; time cells signal the nature and timing of salient events, just as place cells signal the nature and spatial location of salient events; time cells disambiguate overlapping sequence memories, just as place cells disambiguate overlapping routes; and time cells partially "retime" when key temporal parameters are altered, just as place cells partially "remap" when critical spatial cues are altered. These findings suggest that hippocampal neuronal ensembles segment temporally-organized memories much as they segment spatial memories. MacDonald and coworkers (2011, abstract) conclude that "place cells and time cells reflect fundamental mechanisms by which hippocampal neural networks parse any spatiotemporal context into quantal units of where and when important events occur and bridge, and thereby organize elements, in a conceptual organization of events." Eichenbaum (2014, abstract) concludes that "the firing properties of time cells parallel the properties of place cells and provide an additional dimension that is integrated with the spatial dimensions." Eichenbaum (2014) concludes that "hippocampal neurons differentially encode key events in space and time and compose unique spatially and temporally-organized representations of specific experiences. The representation of time and space in the hippocampus is a fundamental mechanism for organizing the elements of experience." Spacetime intervals appear to be the fundamental units through which time and space combine to organize the elements of experience.

\section{Spacetime Intervals}

An event is the fundamental entity of observed physical reality represented by three coordinates of space and one coordinate of time in the 
spacetime continuum postulated by the theory of relativity. A conscious event, considered in relativistic terms, is the fundamental entity of conscious experience (observed physical reality) represented by three coordinates of space and one coordinate of time in the spacetime continuum postulated for conscious experience. In relativistic physics, an event has a unique position specified by four coordinates $(x, y, z, t)$. The unification of space and time is exemplified by the common practice of selecting a metric such that all four dimensions are measured in terms of units of distance $(x, y, z, c t)$, where " $c$ " represents the speed of light in a vacuum and " $c t$ " represents distance along the time axis (Petkov, 2010). Since $c$ is equal to $1, c t$ reduces to $t$. This enables mathematical description of spacetime, the positions of events in spacetime, and most importantly, spacetime intervals.

In three-dimensional space, the separation between two objects is measured by the distance between them (the distance is purely spatial and always positive). In spacetime, the separation between two events is measured by the invariant spacetime interval between the events, which takes into account not only their spatial separation, but their temporal separation as well. Spacetime interval may be formulated as follows: the spacetime interval "s" is equal to the difference between the space coordinates " $\Delta r$ " of two events minus the difference between the time coordinates " $c \Delta t$ " of the two events (in practice the square is utilized as the sign of the spacetime interval " $s$ " is indefinite-positive, negative, or zero; " $r$ " is a displacement vector);

(spacetime interval) $=$

$$
s^{2}=\Delta r^{2}-c^{2} \Delta t^{2} \text { or } s^{2}=\Delta r^{2}-\Delta t^{2}, \text { if } c=1 \text {. }
$$

\section{The Light Cone}

Spacetime intervals are defined by the light cone. A pulse of light emitted from a point spreads outwards in all directions at $c$. If light is confined to a two-dimensional plane, the light from a flash spreads out in a circle (Figure 1 and 2). If the growing circle is drawn with the vertical axis representing time (as in Figure 1 and 2), the result is a light cone (Penrose, 2005). In reality, there are three space dimensions, so light would actually form an expanding sphere and the light cone would be a $4 \mathrm{D}$ version of a cone. The concepts are easier to visualize with the number of space eISSN 1303-5150 dimensions reduced to two. A light cone represents the path that a flash of light, emanating from a single event and travelling at the speed of light in all directions, would take through spacetime. Light cones are the same for all events and for all observers (since $c$ is a universal constant). Given an event $A$, all events that can be reached by a light pulse from $A$ form a future light cone, while all events that can send a light pulse to A form an inverted past light cone (Figure 1 and 2). Three types of spacetime intervals are defined by the light cone and the formulation for spacetime interval. Spacetime intervals play a crucial role in the organization of conscious experiences.

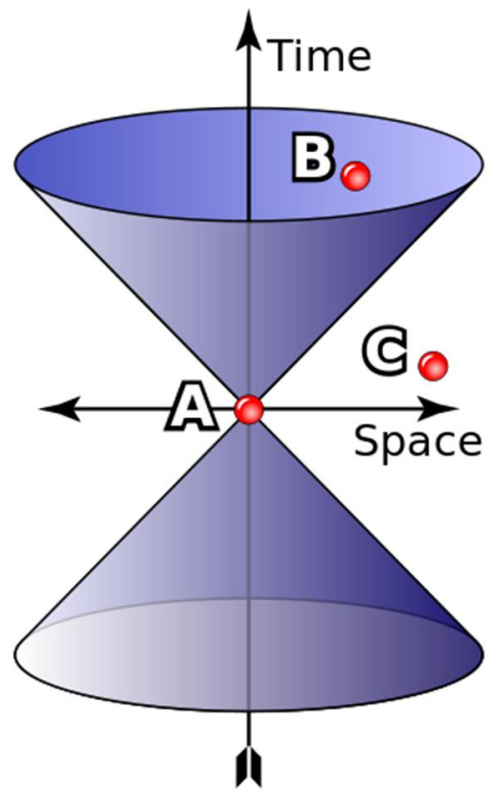

Figure 1. Spacetime Intervals.

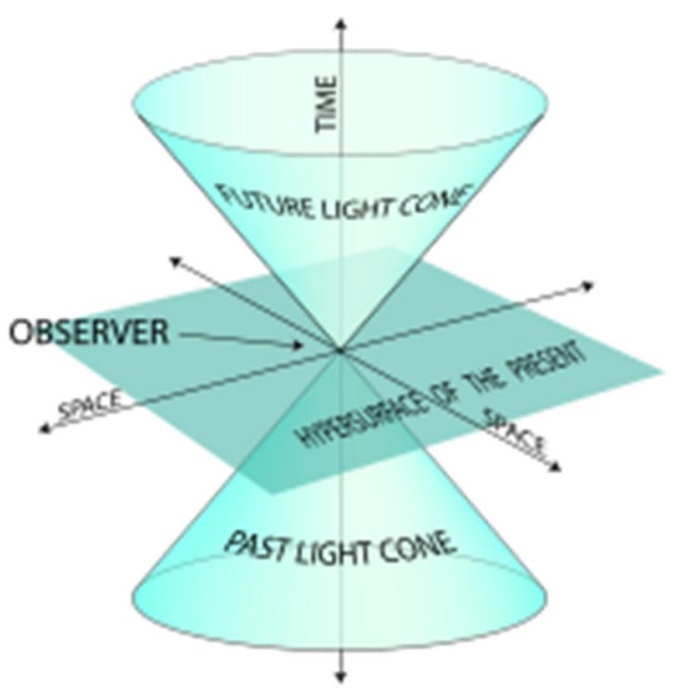

Figure 2. Past and Future Light Cones. 


\section{Light-Like Spacetime Intervals}

Two events on a light cone are separated at $c$. This is a light-like spacetime interval. The difference in the space coordinates of two events on a light cone is exactly equal to the difference in the time coordinates of the events $\left(\Delta r^{2}=\Delta t^{2}, s^{2}=0\right.$, see Figure 1). There is a reference frame where events are separated at $c$ (events occurring to a photon as it travels along its path). Such occurs when you directly observe a light source or any event (the event is visible because of reflected light). Lightlike spacetime intervals may help determine the nature of conscious events by carrying the defining information about the events to the eyes.

\section{Time-Like Spacetime Intervals}

If the difference in the time coordinates of two events is greater than the difference in the space coordinates of the events $\left(\Delta t^{2}>\Delta r^{2}\right)$, the events fall inside a light cone (A and $B$ in Figure 1 ) and the separation is called a time-like spacetime interval. There is a reference frame where two events with time-like separation may be observed at the same spatial position, but not at the same time. The events may be observed at the same spatial position, but at different times (the events are separated only by time). Time-like spacetime intervals could account for the observation (experience) of conscious events at the same spatial positions, but at different times (this can occur only with time-like separation of the events).

\section{Space-Like Spacetime Intervals}

If the difference in the space coordinates of two events is greater than the difference in the time coordinates of the events $\left(\Delta r^{2}>\Delta t^{2}\right)$, the events fall outside a light cone (A and $\mathrm{C}$ in Figure 1 ) and the separation is called a space-like spacetime interval. There is a reference frame where two events with space-like separation may be observed at the same time, but not at the same spatial position. The events may be observed simultaneously, but at different spatial positions (the events are separated only by space). Spacelike spacetime intervals could account for the observation (experience) of conscious events simultaneously, but at different spatial positions (this can occur only with space-like separation of the events).

\section{Organization of Conscious Experience}

Spacetime intervals account for the organization of conscious experience. Spacetime intervals in effect link (associate) conscious events to form a coherent four-dimensional conscious experience (ie. a coherent four-dimensional observation of conscious events). Spacetime intervals explain why conscious experience appears to us the way it does. Conscious experience is essentially an orientation in space and time (an awareness of the existing situation with reference to space, time, and identity). It is an understanding of the position of the observer in space and time.

\section{Representation of Spacetime Intervals}

In 2014, John O'Keefe, May-Britt Moser, and Edvard Moser were awarded the Nobel Prize in Physiology or Medicine for the discovery of "grid cells," cells that constitute a "positioning system" in the brain. A grid cell is a type of neuron found in the brains of many species that allows them to "understand" their position in space (Hafting et al., 2005; Doeller et al., 2010; Sieb, 2016). Grid cells have been identified in rats, bats, monkeys, and humans. A grid cell fires when a freely-moving animal traverses a set of small regions (firing fields) roughly equal in size and arranged in a periodic triangular array that covers the entire available environment (Hafting et al., 2005). Firing fields are equally spaced apart, such that the distance from one firing field to all six adjacent firing fields is approximately the same. Firing fields are positioned such that the six neighboring fields are located at approximately 60 degree increments (firing fields are organized into a hexagonal lattice, Figure 3). Doeller and coworkers (2010) provided the first evidence for grid-cell-like representations in humans that implicates a network of regions supporting spatial cognition and autobiographical (episodic) memory. This network consisted of the entorhinal/hippocampal/subicular, posterior and medial parietal, lateral temporal, and medial prefrontal cortical areas. The signal was greatest in the right entorhinal cortex. Doeller (abstract) concluded that "grid cells provide a strikingly periodic representation which is suggestive of very specific computational mechanisms." 


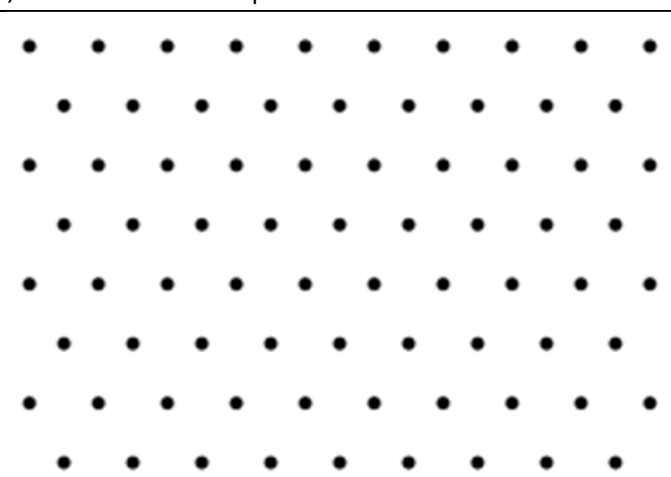

Figure 3. A hexagonal lattice. The dots are firing fields of a grid cell.

The entorhinal cortex has reciprocal connections with the hippocampus and many other brain areas (Serino and Riva, 2014). A number of connections are important in hippocampal function. Besides output to the entorhinal cortex, additional output goes to other cortical areas, including the prefrontal cortex (PFC), posterior parietal cortex (PPC), lateral septal area, and mammillary body. A key function of PPC is the implementation of visuospatial attention and of spatial processing in general (Wendelken, 2015). The intraparietal sulcus (IPS), which separates the inferior and superior parietal lobes (IPL, SPL), has been shown to contribute to the maintenance of spatial location information and the IPL is a locus of spatialrelational processing. Selectivity for higher-order visuospatial processing was observed in right PPC. Lara and Wallis (2015) found that the strongest activity of the overwhelming majority of PFC neurons reflect the spatial location of stimuli and the passage of time. The PPC and PFC areas have been implicated in time-experiencing (Wendelken, 2015): the cerebellum, right PPC, right $\mathrm{PFC}$, fronto-striatal circuits, and insular cortex for duration perception; the inferior frontal and superior temporal lobes, hippocampus, medial PFC, medial parietal and posterior cingulate cortex for past-future distinction and mental time travel; the PFC, IPC, superior colliculus, and insular cortex for synchronous and asynchronous event distinction; and the posterior sylvian regions, PPC, and temporo-parietal networks for temporal order judgment. Wendelken (2015) observed stronger PPC activation for processing inequalities, than for processing equalities, and argued that this was due to representation of the more specific inequality relationships in PPC. This is interesting because space-like and time-like spacetime eISSN 1303-5150 intervals are inequalities. Wendelken (2015) found that current evidence is most consistent with accounts that involve estimation and probabilistic computation for the PPC and PFC. PPC is a primary area for mathematical (numerical) cognition. Evidence from a largescale meta-analysis indicated clearly that the pattern of activation of PPC is associated most closely to mathematical cognition (Wendelken, 2015). In addition to these parietal regions, regions of the frontal lobe are also active in calculation tasks. In monkeys, neurons have been found in the frontal cortex and in the IPL that respond to numbers (Wendelken, 2015). There is evidence that numerical cognition is intimately related to spatial cognition (Wendelken, 2015). Regions of the parietal cortex show shared activation for both spatial and numerical processing, and as mentioned above, for temporal processing. These various lines of research suggest a strong, but flexible, connection between numerical, spatial, and temporal processing in PPC and PFC. Wendelken (2015) found that the extraction of mental relations from the hippocampus by the PPC and PFC initiates an important process of relational integration. These studies support the contention that the PPC and PFC may preferentially extract spacetime interval inequality information from the hippocampus in a process of relational integration (Sieb, 2016).

The IPC contains neurons which are multimodally responsive, receiving highly processed input from somesthetic, visual, and auditory association cortex, the frontal lobes, and other higher-order assimilation areas throughout the neocortex (Joseph, 1990, 2000; Sieb, 2013). All higher-order sensory processing converges on the IPC. IPC neurons can simultaneously analyze visual, auditory, and somesthetic information, respond to visual stimuli of any size, shape, or form, and have visual receptive properties that span almost the entire visual field. The IPC is involved in the creation and assimilation of cross modal associations (auditory, visual, and somesthetic equivalents of objects, events, ideas, actions, feelings). The IPC appears to be involved in perception. Perception is shaped by learning, memory, expectation, and attention (Bernstein, 2010; Gregory, 1987), processes which the hippocampus, PPC (including the IPC), and PFC are engaged in (Sieb, 2013, 2015). Perception also has features which may be conditioned by the hippocampus-PPC-PFC: constancy (the ability to recognize the same event from widely varying 
sensory inputs-Atkinson et al., 1990; Bernstein, 2010), grouping (humans naturally perceive events as organized sets of qualia-Goldstein, 2009; Gray, 2006; Wolfe et al., 2008), contrast (qualia can be affected by the qualities of context-Corsini, 2002; Kushner, 2008; Popper, 2010), experience (with experience, organisms can make finer perceptual distinctions and learn new kinds of categorization-Sumner,2009), motivation (Coon and Mitterer, 2008; Sieb, 2013; Weiten, 2010), and expectation (a predisposition to perceive things in a certain way-Coon and Mitterer, 2008; Sieb, 2013; Weiten, 2010). Perception appears to involve the hippocampus-PPC-PFC (Sieb, 2013). Since conscious experience is such an intimate part of perception, the hippocampus-PPC-PFC is probably also involved in the organization of conscious experiences.

Reasoning (the capacity to reach novel conclusions on the basis of existing premises) is among the most complex of cognitive processes (Wendelken, 2015). Bilateral PPC activation was found during relational reasoning and left PPC activation during propositional reasoning. Within PPC, reasoning is most strongly associated with activation of middle to posterior IPL, and to a lesser extent, with neighboring regions of SPL. Left PPC demonstrated greater involvement than right PPC. Selectivity for higher-order visuospatial reasoning, but not semantic reasoning, was found in right PPC. There were notable similarities between reasoning activations and activations associated with visuospatial processing and attention, particularly on the right, and between reasoning and phonological processing, particularly on the left. The mid-IPL appears to be unique for relational reasoning. Wendelken (2015) observed stronger PPC activation for reasoning with inequalities, than for reasoning with equalities and that current evidence points away from logical rule-following as a primary mechanism for reasoning and is more consistent with accounts that involve estimation and probabilistic computation. He found that it was clearly indicated that the pattern of activation in PPC associated with reasoning is most closely related to that for mathematical cognition. Wendelken (2015) found that rostrolateral PFC extracts mental relations from the hippocampus and is specialized for second-order relational reasoning. He found that current results are consistent with the possibility that rostrolateral PFC may share this duty with a sub-region of midIPL. Although direct anatomical connections between rostrolateral PFC and mid-IPL have not been reported, it is noteworthy that these two regions demonstrate strong functional connectivity during task execution and even at rest. The PPC and PFC also contribute to episodic memory. Parietal activation is most commonly associated with the endorsement of stimuli as having been previously encountered (Nelson et al., 2013; Wagner et al., 2005), though associations with memory encoding (Uncapher and Wagner, 2009) and memory confidence (Johnson et al., 2013) have also been noted. The PFC (particularly in the left hemisphere) is involved in the formation of new episodic memories. Patients with damage to the PFC can learn new information, but tend to do so in a disordered fashion (Janowski et al., 1989). The PFC may be essential for remembering the contextual details of episodic memory, help organize information for more efficient storage, or underlie semantic strategies which enhance encoding (Gabriele and Kao, 2007; Gabriele et al., 1998). Hence the PPC and PFC are intimately involved in complex cognitive functioning (perception, reasoning, episodic memory, mathematical cognition, spatial and temporal cognition) and spacetime interval inequalities extracted from the hippocampus may play a key role in this processing.

\section{Causality}

Because signals and other causal influences cannot travel faster than the speed of light, light cones (Figure 1 and 2) define the concept of causality. For time-like spacetime intervals (inside light cones), there is enough time between the events that signals or information can travel between the events at less than the speed of light. Hence one event can influence or be influenced by the other event, by signals or information that does not need to travel faster than the speed of light. One event could be the cause or effect of the other event. If $B$ causes $A, B$ exists in the past history (in the past light cone) of A (Figure 1 and 2 ). If $B$ is caused by $A, B$ exists in the future (in the future light cone) of A (Figure 1 and 2). The past light cone of an event represents the boundary of its causal past and the future light cone the boundary of its causal future. Events with timelike spacetime interval separation may be said to have a past or future relation.

When a space-like spacetime interval separates two events (outside light cones), not enough time passes between their occurrences for 
there to exist a cause-effect relationship crossing the spatial distance between the events at the speed of light or slower. Generally, the events are considered not to have a past-future relation.

Causality is distinct from mere contingency or covariation (Cummins, 2014). In causality, one event has the power to bring about another event. In covariation and contingency, two events are simply statistically dependent on one another. Neuro-imaging studies show that the brain distinguishes causal events from non-causal events (Cummins, 2014). There are significantly higher relative levels of activation in the right middle frontal gyrus and the right IPL for causal relative to non-causal events. Causal judgments, beyond associative judgments, generated distinct activation in left dorsolateral PFC and right precuneus (part of the SPL), substantiating the particular involvement of these areas in assessments of causality. Perceptual causality can be distinguished from inferential causality (Cummins, 2014). Inferential causality activates the medial frontal cortex, with particular left hemispheric involvement. Perceptual causality activates the right parietal lobe suggesting that the right parietal lobe is involved in the processing of the spatial attributes of causality. The PPC and PFC therefore appear to be involved in the assessment of causality. Since they also appear to be involved in the processing of spacetime interval inequalities, assessments of causality could arise in conjunction with the processing of spacetime interval inequalities by the PPC and PFC. This supports the contention that spacetime interval inequalities are represented in the hippocampus and extracted by the PPC and PFC for perception, the organization of conscious experience, and the integration of complex cognitive functions. Causal inference is a fundamental component of cognition and perception, binding together conceptual categories, imposing structures on perceived events, and guiding decision-making (Cummins, 2014).

\section{Working Memory}

The concept of working memory describes a process of short-term storage of information to support ongoing or upcoming actions, and is considered a crucial component of the executive control of goal-directed behavior (Mansouri et al., 2015). The retention of task-relevant information is essential for complex behaviors which evolve in time, in order to maintain the perception and actions in a coherent and goal-directed framework. Working memory is crucial for the temporal organization of behavior, linking processes across delays. Working memory is considered an essential intermediate stage enabling further manipulation and integration of information involved in perceptual and mental functions. Functionally, working memory may be considered the provisional retention of perceptual information for prospective action, a type of focal attention whereby perception is reorganized and re-represented, becoming explicit, functional, and conscious (Sieb, 2004, 2015, 2016). Neural correlates of working memory have been found in many different brain areas, including those typically involved in perceptual and motor functions (Mansouri et al., 2015). Study has supported the idea that working memory is based on maintained representation of events and stimuli, even after their cessation, in PFC neuro circuitry. It was suggested that such representations enable temporal linking of recent salient experiences to the upcoming action. Cellular activity in other cortical areas, particularly the PPC, also conveys information during delay periods. Different research groups found sustained neuronal activity in delayed response tasks in various compartments of the PFC, as well as in the sensory and motor areas (Mansouri et al., 2015). These studies showed that, depending on the task demand, information about different stimulus features, from different modalities, could be represented and maintained in neuronal activity within the PFC and posterior sensory areas. Working memory maintains abstract information, such as number, location, time, color, and shape. A number of features have been described for working memory (Mansouri et al., 2015): it has a short duration and fades as the delay period gets longer; it is goal-oriented and its content is used to guide upcoming behavior; it is limited to a trial, being updated in each subsequent trial; it is highly vulnerable to distraction; its content is a discrete feature of an object or event, such as a particular color, shape, position, or time in space. Subjects intentionally store information in working memory to solve a problem and are therefore aware of, and consciously experiencing, its content.

A widely held view of PFC function is that it encodes task relevant information in working memory (Lara and Wallis, 2015). This position originates from decades of work showing strong neural activity in PFC during the delay period of 
working memory tasks. This delay period activity has two key properties (Lara and Wallis, 2015): it is specific to the stimulus being remembered (consistent with it containing information about the content of working memory) and it only encodes stimuli that are relevant to the task at hand (it is resistant to distractors and task irrelevant information is not encoded in working memory). In recent years there has been a steady stream of novel research that has challenged the widely held view that PFC stores task relevant information in working memory. Lara and Wallis (2015) found that the overwhelming majority of PFC neurons failed to encode working memory; instead, the strongest signals reflected the passage of time and the spatial location of the stimuli. Both of these signals could play an important role in organizing behavior towards the performance of the task, but they do not reflect the contents of working memory. They also support the contention that the PFC processes spacetime interval inequality information for cognition and conscious experience. Lara and Wallis (2015) suggested that the PFC neurons appear to have encoded attentional control signals that helped improve performance. This view suggests that sensory information is maintained in working memory by the same sensory neurons that represent that information when it is present in the sensory environment and there is strong evidence that posterior sensory areas (including the PPC) play a strong role in the storage of information in working memory. Hence the role of PFC is not to store information in working memory, but rather to actively focus attention on the relevant sensory representation, select information, and perform executive functions that are necessary to control the cognitive processing of the information. Hence, in accordance with the premise of this paper, the PFC appears to use spacetime interval inequality information to control cognitive processing.

Working memory then is the holding in mind of multiple pieces of information for a brief period of time and its manipulation. Working memory has been linked to attention, conscious experience, learning, cognitive development, cognitive function, and memory (Lara and Wallis, 2015; Sieb, 2015, 2016). The PFC, PPC, thalamus, and parts of the basal ganglia (caudate, globus pallidus) are crucial for working memory function (Sieb, 2013, 2015, 2016). There is an emerging consensus that most working memory tasks recruit a network of prefrontal and parietal cortical areas. Since the PPC and PFC appear to integrate spacetime interval inequalities extracted from the hippocampus, spacetime interval inequalities could play an important role in the functions of working memory. An increasingly large number of investigations have found that the feedback connectivity between the PFC and PPC is required for working memory function and conscious experience. Positive feedback appears to be involved (Sieb, 2004, 2011, 2013, 2015).

\section{Theta}

The medial septal area sends cholinergic and GABAergic fibers to all parts of the hippocampus. These play a key role in controlling the physiological state of the hippocampus; destruction of the septal area abolishes the hippocampal theta rhythm and severely impairs certain types of memory. Neural activity in nearly every part of the hippocampal system is modulated by theta. The entorhinal cortex is no exception. Like the hippocampus, it receives cholinergic and GABAergic input from the medial septal area, the central controller of theta. Grid cells, like hippocampal place cells, show strong theta modulation (Hafting et al., 2005). The theta mode appears in the hippocampus during states of active, alert behavior and also during REM sleep. When cortical desynchronization occurs (during active searching, orientation, maintained and selective attention, initial stages of learning, discrimination responses, initial exposure to novel stimuli), hippocampal theta appears (Buzsaki, 2006); that is, theta appears in the hippocampus whenever an animal is actively engaged with its environment. The theta rhythm reflects subthreshold membrane potentials which strongly modulate the spiking of hippocampal neurons and synchronise across the hippocampus in a travelling wave pattern (Lubenov and Siapas, 2009).

Frontal-midline ( $\mathrm{fm}$ ) theta oscillations are of particular interest in regard to higher cognitive functions (Enriquez-Geppert et al., 2014). Fmtheta oscillations are recorded over fronto-medial brain regions at frequencies between $4-8 \mathrm{~Hz}$ and appear to be generated in the mid-cingulate cortex (MCC), a highly interconnected brain structure, that is part of the superordinate cognitive control network. The MCC is known to be crucially involved in executive functioning, which enables goal-directed behavior. Enhanced cognitive processing is accompanied with increases of fm- 
theta, specifically in tasks involving working memory and executive functions. In addition, fmtheta activity has been related to efficient working memory maintenance and increases of fm-theta activity during task processing have been shown to predict successful behavioral performance and conflict monitoring.

In addition to local synchronization, oscillatory activity of distant neural structures can synchronize (Enriquez-Geppert et al., 2014; Lara and Wallis, 2015). Because brain areas are bidirectionally coupled, the connections between brain areas form feedback loops. Oscillatory activity generally arises from feedback connections that result in the synchronization of firing patterns; positive feedback loops tend to cause oscillatory activity in which frequency is inversely related to the delay time. Oscillations from multiple cortical areas can become synchronized to form a large-scale oscillating network. Neural oscillations may coordinate neuronal spiking between and within brain circuits and coherent large-scale brain activity may form dynamic links between brain areas required for the integration of distributed information. Neural oscillations may provide a linkage of neural activity with behavior and thought. Different neural oscillations may appear concurrently and interact in a hierarchical way in order to implement perception and cognition. Synchronization and neural oscillation have been linked to many cognitive functions such as information transfer, perception, motor control, and memory. Neural oscillations have been linked to cognitive states, such as awareness and consciousness (Enriquez-Geppert et al., 2014). Hence theta oscillations appear to be correlated with the operation of working memory and the information transfer involved in perception, cognitive processing, learning, memory, motor control, and conscious experience. Spacetime interval inequality relations may be distributed and integrated throughout a wide spread thetaoscillating hippocampus-PPC-PFC network to condition this processing (Sieb, 2016). Relativistic spacetime intervals in effect compose and direct our conscious life.

Quantum Mechanics, Classical Physics, and Conscious Experience

Classical physics refers to those physical theories which do not include the quantisation paradigm: classical mechanics (Newton's Laws of Motion, classical Lagrangian and Hamiltonian formalisms), classical electrodynamics (Maxwell's Equations), classical thermodynamics, special relativity, general relativity, classical chaos theory, and nonlinear dynamics (Morin, 2008). Quantum refers to the minimum amount of any physical entity involved in an interaction; the smallest amount of many forms of energy; certain characteristics of matter which can take only discrete values. Quantum mechanics is highly successful in describing microscopic objects (atoms, molecules, elementary particles) and their interactions. Physical objects, ranging from those larger than atoms and molecules, to objects in the macroscopic and astronomical realm, can be welldescribed with classical physics; macroscopic or large systems are accurately described by classical theories (Jaeger, 2014). Beginning at the atomic level and lower, the laws of classical physics break down and generally do not provide a correct description of nature. As a system becomes larger or more massive, classical dynamics tends to emerge.

If quantum mechanics were to be applicable to macroscopic objects, there must be some zone of applicability (limit) in which quantum mechanics reduces to classical mechanics (Bohr's Correspondence Principle). The conditions under which quantum mechanics and classical physics agree are referred to as the correspondence or classical limit. Bohr provided a rough prescription for the classical limit: it occurs when the quantum numbers describing the system are large, ie. classical physics and quantum physics give the same answer when the systems become large (Bohr, 1976; Jaeger, 2014; Tipler and Llewellyn, 2008). As part of the Copenhagen interpretation of quantum mechanics, it was generally accepted that the quantum mechanical description of large systems should closely approximate the classical description. A way of making a quantum system large is to represent it in conscious experience. Whenever we describe a quantum system, we describe it in conscious experience. Whenever we describe a classical system, we also describe it in conscious experience. Conscious experience is where quantum systems and classical systems agree. When we represent a quantum system in conscious experience, we represent the system as a large (macroscopic) system and use classical physics to describe it. Hence we make a quantum system "large" by representing it in conscious experience. We can usually ignore quantum 
mechanics in our every day description of large systems and the classical description will suffice. Hence conscious experience may be considered the classical or correspondence limit in which quantum mechanics is reduced to classical physics.

The reduction of quantum mechanics to classical physics can be detected in everyday conscious experience. Vision accounts for much of our conscious experience. Wave-particle duality is a quantum mechanical feature of light: light travels in some respects like a particle (discrete units, called photons, which predict experienced energies, colors, and spectral intensities; light is reflected) and in some respects like a wave (experienced color is a function of wavelength, experienced intensity is a function of frequency, light can be reflected or diffracted). The waveparticle duality of light yields the conscious experience of macroscopic colors, intensities, shapes, objects, locations, and times. The experienced world is well-described by classical physics (measurement, etc.). Hence our visual system transforms a quantum mechanical feature into the classical physics of conscious experience; wave-particle duality is reduced to classical physics in conscious experience. Conscious experience is the classical limit for wave-particle duality.

Building on de Broglie's hypothesis (all matter has a wave-particle duality), the quantum wave function was developed. The quantum wave function describes the totality of a quantum system. Erwin Schrödinger developed an equation that describes the behavior of a quantum mechanical wave. The Schrödinger equation is central to quantum mechanics and describes how the quantum state of a physical system changes in time. It defines the permitted stationary states of a quantum system and provides a means of predicting the probability of measurement results. Schrödinger's equation provides the means for predicting conscious experiences which may evolve from a quantum system.

Whenever we represent and describe a quantum system, we represent it in conscious experience. The uncertainty principle, for example, dictates that the more you know about one aspect of a fundamental particle, such as its position, the less you can know about another related aspect of that particle, such as its momentum-and vice versa (Hossenfelder, 2015). Observation or measurement of one aspect

eISSN $1303-5150$ of the particle reduces information of other related aspects of that particle. The entire phenomenon is represented and described in conscious experience (and a quantum system may be thought of as reduced to classical physics in conscious experience). The paradoxical behavior of "entangled" particles (pairs of particles that share a common quantum state) unfolds as follows (Hossenfelder, 2015): imagine (conscious experience) an unstable particle with a spin of zero decaying into two daughter particles, which speed-off in opposite directions. Conservation laws dictate that the spins of those two daughter particles must add up to zero; one particle, then, could possess a spin value of "up," and the other could have a spin value of "down." The laws of quantum mechanics dictate that neither of the particles possesses a definite spin until one of the two speeding entangled particles is measured (that is, represented in conscious experience). The whole phenomenon unfolds in conscious experience (just reading the preceding description manifests the entire quantum phenomenon in conscious experience). In effect, the whole phenomenon is deduced through conscious experience. All quantum phenomena similarly are deduced through conscious experience.

Einstein used his imaginings (thought experiments) to make major breakthroughs and his greatest discoveries (Hossenfelder, 2015). As just described, imaginings also play a huge role in representing and describing quantum phenomena. Special relativity, general relativity, atomic theory, quantum mechanics, Newton's laws of motion, and many other theories grew out of such imaginings. Imaginings are conscious experiences. All observed phenomena are represented and described through conscious experience. All observed phenomena are deduced through conscious experience. The genius of Einstein and others (Aristotle, Galileo, Newton, Schrödinger, etc.) was in realizing which aspects of experience were essential and which could be discarded.

All observed physical phenomena then are deduced through conscious experience. With his special theory of relativity, Einstein showed that space and time do not have independent existences but instead form a fabric of spacetime (Isaacson, 2015). Einstein's general theory of relativity showed that this fabric of spacetime was not merely a container for events, but had its own dynamics, that were determined by, and in turn 
helped to determine, the movements of objects within it (Isaacson, 2015). It is interesting that special relativity and general relativity were themselves deduced through conscious experience. They essentially model the conscious experience from which they were deduced. What Einstein appears to have discovered with his theories of relativity is the organizational framework of human conscious experience. Earlier, it was shown how spacetime intervals organize coherent conscious experiences. Spacetime intervals are fundamentally involved in the organization of all conscious experiences.

\section{Theory of Everything (ToE)}

Finding a ToE is one of the major unsolved problems in physics. A ToE is a hypothetical single, all-encompassing, coherent theoretical framework of physics that fully explains and links together all physical aspects of the universe (Weinberg, 1993). Since all physical aspects of the universe are represented and described only through conscious experience, a ToE must fully explain and link together all conscious experience. The ToE would be expected to be based on axioms from which all observable physical phenomena (all conscious experiences) are deduced. Since spacetime intervals are responsible for the organization of all coherent conscious experiences (all observable physical phenomena), the equation formulating spacetime intervals may be thought of as the axiom from which all conscious experiences (and therefore all observable physical phenomena of the universe) may be deduced. $s^{2}=\Delta r^{2}-\Delta t^{2}$ therefore may be considered the fundamental equation, the ToE. $s^{2}=\Delta r^{2}-\Delta t^{2}$ is a single, allencompassing, coherent theoretical framework of physics which fully explains and links together all physical aspects of the universe (all observable physical phenomena, all conscious experiences).

Over the past few centuries, two theoretical frameworks have been developed that, as a whole, most closely resemble a ToE (Carlip, 2001; Priest, 2010). The two theories upon which all modern physics rests is general relativity (GR) and quantum field theory (QFT). GR is a theoretical framework that focuses on the force of gravity for understanding the universe in regions of both large-scale and high-mass (stars, galaxies, clusters of galaxies, etc.). QFT is a theoretical framework that focuses on three nongravitational forces (strong, weak, electromagnetic) for understanding the universe eISSN 1303-5150 in regions of both small scale and low mass (atoms, molecules, sub-atomic particles, etc.). Physicists have experimentally confirmed with tremendous accuracy virtually every prediction made by these two theories when in their appropriate domains of applicability. Scientists also have learned that GR and QFT, as they are currently formulated, are mutually incompatiblethey cannot both be right. Since the usual domains of applicability of GR and QFT are so different, most situations require that only one of the two theories be used. As it turns out, this incompatibility between GR and QFT is only an apparent issue in regions of extremely small-scale and high-mass, such as those that exist within a black hole or during the beginning stages of the universe (ie. the moment immediately following the Big Bang). To resolve this conflict, a theoretical framework revealing a deeper underlying reality, unifying gravity with the other three fundamental interactions, must be discovered to harmoniously integrate the realms of GR and QFT into a seamless whole: a single theory that, in principle, is capable of describing all phenomena. The relativistic theory of consciousness appears to accomplish this. Every observed phenomenon, whether it is described in GR or QFT, we know and will know, is represented and described through conscious experience. Conscious experience essentially weds the smallscale world of quantum mechanics with the largescale world of classical physics. It harmoniously integrates GR and QFT into a seamless whole. Future breakthroughs in physics (such as the origin of the universe, the nature of life) will eventually be represented and described through conscious experience. The potentiality of this representation exists in $s^{2}=\Delta r^{2}-\Delta t^{2}$. It just has to be extracted. That is why $s^{2}=\Delta r^{2}-\Delta t^{2}$ is the ToE, it explains everything.

\section{Conclusion}

Human conscious experience is four-dimensional. The neural correlate for four-dimensional conscious experience has been found in the human brain and is modeled by Einstein's special theory of relativity. A representation of key events in space and time is encoded in the hippocampus and stored as episodic memory. Entorhinal cortex grid cells establish a reference grid in the hippocampus for the computation of spacetime intervals. Light-like spacetime intervals establish conscious events. Space-like and time- 
like spacetime intervals (spacetime interval inequalities) establish the relative positions of conscious events in conscious experience. Spacetime intervals in effect link (associate) conscious events to organize coherent conscious experiences. Spacetime intervals account for why conscious experience appears to us the way it does. Conscious experience is an orientation in space and time (spacetime), an understanding of the position of the observer in space and time (spacetime). The posterior parietal cortex and prefrontal cortex extract spacetime interval relations from the hippocampus for perception and the organization of conscious experiences, the assessment of causality and past-future relationships, the integration of higher cognitive functions, learning, episodic memory, and the implementation of goal-directed actions. This occurs via a widespread theta-oscillating entorhinal-hippocampal-posterior parietalprefrontal cortical network known as working memory.

The relativistic concept of conscious experience has great explanatory power when it comes to consciousness. It closes the "explanatory gap" and solves the "hard problem of consciousness" (how can something subjective like conscious experience arise in something physical like the brain). Consciousness arises from sensory input and is completely physical, confined to, and dependent on the physical brain. Consciousness can no longer be considered mysterious, non-physical, epiphenomenal, or indescribable. It has a definite location and functions-attention, perception, formation of conscious experiences, assessment of causality and past-future relations, learning, memory, cognitive function, and implementation of goaldirected actions. This concept explains why conscious experience appears to us the way it does. I can think of no other way to explain this. It has been said (Koch, 2015, p. 31) that "nowhere in physics does consciousness appear, but the brain is a physical object and has consciousness." From the proceeding, it was shown that consciousness does have a place in physics. Conscious experience not only has a relativistic framework and explanation, but is the medium from which all observable phenomena are deduced. All phenomena, irrespective of what level they are observed or described at, are known to us only through conscious experience. All future phenomena, even those describing the origin of the universe and of life itself, will eventually be described in some way through conscious experience. Since spacetime intervals direct the formation of all conscious experiences, the equation formulating spacetime intervals potentially contains the information from which all observable phenomena, and future physical phenomena, may be deduced. It is therefore the fundamental equation, the "Theory of Everything."

One application of the above principles might be in the amelioration of psychological stress and of psychiatric conditions. For example, a person might like to go swimming regularly. Beforehand, the person may imagine the complete swimming experience: difficulties getting to the pool, nasty patrons at the pool, poor or uncertain pool conditions and scheduling, finding a suitable swimming lane, pain and stiffness before swimming, pain and fatigue during swimming, recovery after swimming, difficulties returning home. If a person experiences all these conscious events simultaneously beforehand (the conscious events have space-like separation in the experience) the swimming experience may become very stressful and even overwhelming (the stress may be compounded by experiencing all the events simultaneously), and the person may even stay home. However, if the person dispenses with this space-like experience and instead experiences each event separately as it comes up (time-like experience of conscious events), the overall swimming experience may be considerably less stressful and may even be pleasant. If the person can learn to experience stressful conscious events individually and separately, and not simultaneously, stress and anxiety may be reduced or avoided. Much of the distress of depression and anxiety conditions might arise from such space-like rumination of distressful conscious events. If the patients could replace this with a time-like approach (considering each event separately, one at a time), it might help ameliorate some psychiatric conditions. 


\section{References}

Atkinson RL, Atkinson RC and Smith EE. Introduction to Psychology. Harcourt Brace Jovanovich 1990; 177-183.

Bernstein DA. Essentials of Psychology. Cengage Learning 2010; 123-124.

Bohr N. Niels Bohr, Collected Works, Vol.3, The Correspondence Principle (1918-1923). North-Holland, Amsterdam 1976.

Buzsáki G. Rhythms of the Brain. Oxford University Press 2006.

Carlip S. Quantum gravity: A progress report. Reports on Progress in Physics 2001; 64(8): 885.

Coon D and Mitterer JO. Introduction to Psychology: Gateways to Mind and Behavior. Cengage Learning 2008; 171-172.

Corsini RJ. The Dictionary of Psychology. Psychology Press 2002; 219.

Cummins DD. Neural correlates of causal power judgments. Frontiers of Human Neuroscience 2014; 8: 1014. doi: 10.3389/fnhum.2014.01014

Disalle R. Space and Time: Inertial Frames. The Stanford Encyclopedia of Philosophy (Winter, 2009 Edition), E.N. Zalta (Ed.) 2009.

Doeller CF, Barry C and Burgess N. Evidence for grid cells in a human memory network. Nature 2010; 463\{7281\}: 657661.

Eichenbaum $H$. Time cells in the hippocampus: a new dimension for mapping memories. Nature Reviews Neuroscience 2014; 15: 732-744.

Einstein A. Relativity: The Special and the General Theory (Reprint of 1920 translation by Robert W. Lawson), Routledge 2001.

Enriquez-Geppert S, Huster RJ, Figge C and Herrmann CS. Selfregulation of frontal-midline theta memory updating and mental set shifting. Frontiers; 2014; 8: 420. doi: 10.3389/fnbeh.2014.00420

Feynman RP. Six Not-So-Easy Pieces: Einstein's Relativity, Symmetry, and Space-Time. Basic Books, New York 1998.

Gabriele JD and Kao Y. Development of the declarative memory system in the human brain. Nature Neuroscience 2007; 10: 1198-1205.

Gabriele JD and Desmond JE. The role of left prefrontal cortex in learning and memory. Proceedings of the National Academy of Science USA 1998; 95(3): 906-913.

Goldstein EB. Sensation and Perception. Cengage Learning 2009.

Gray PO. Psychology, 5th ed.. Worth, New York 2006: 281.

Gregary R. Oxford Companion to the Mind. Oxford 1987; 598601.

Hafting T, Fyhn M, Molden S, Moser M-B and Moser EI. Microstructure of a spatial map in the entorhinal cortex. Nature 2005; 436(7052): 801-806.

Hossenfelder S. Lost in thought. How important to physics was Einstein's imaginings? Scientific American 2015; 313(3).

Isaacson W. How Einstein discovered general relativity amid war, divorce and rivalry. Scientific American 2015; 313(3).

Jaeger G. What in the (quantum) world is macroscopic? American Journal of Physics 2014; 82(9); 896-905.

Janowski JS, Shimamura AP and Squire LT. Source memory impairment in patients with frontal lobe lesions. Neuropsychologia 1989; 27(8): 1043-1056.

Jeffery K. Integration of sensory inputs to place cells: What, where, why, and how? Hippocampus 2007; 17(9): 775785.

Jeffery K, Anderson M, Hayman R and Chakraborty S. A proposed architecture for the neural representation of spatial context. Neuroscience and Behavioral Reviews 2003; 28: 201-218.

eISSN 1303-5150
Johnson JD, Suzuki M and Rugg MD. Recollection, familiarity and content sensitivity in lateral parietal cortex: A highresolution fMRI study. Frontiers of Human Neuroscience 2013; 7: 209. doi: 10.3389/fnhum.2013.00219

Joseph R. Neuropsychiatry, Neuropsychology, Clinical Neuroscience. Academic Press, New York 1990, 2000.

Koch C. Two natural philosophers, centuries apart, converse about the mind. Scientific American Mind 2015; 26(2): 2831.

Kushner LH. Contrast in judgements of mental health. ProQuest 2008; 1.

Lara $\mathrm{AH}$ and Wallis JD. The role of prefrontal cortex in working memory: A mini review. Frontiers in Systems Neuroscience 2015; 9(173). doi: $10.3389 /$ fnsys.2015.00173

Lubenov EV and Siapas AG. Hippocampal theta oscillations are travelling waves. Nature 2009; 459(7246): 534.

Lucas JR and Hodgson PE. Space, Time and Causality: An Essay in Natural Philosophy, Oxford University Press 1985.

MacDonald CJ, Lepage KQ, Eden UT and Eichenbaum H. Hippocampal "time cells" bridge the gap in memory for discontiguous events. Neuron 2011; 71: 737-749.

Maniadakis $\mathrm{M}$ and Trahanias P. Temporal cognition: A key ingredient of intelligent systems. Frontiers in Neurorobotics 2011; 5(2). doi: 10.3389/fnbot.2011.00002

Mansouri FA, Rosa MGB and Atapour N. Working memory in the service of executive control functions. Frontiers in Systems Neuroscience 2015; 9(166). doi: $10.3389 /$ fnsys.2015.00166

Maurer AP, Vanrhoads SR, Sutherland GR, Lipa P and McNaughton BL. Self-motion and the origin of differential spatial scaling along the septo-temporal axis of the hippocampus. Hippocampus 2005; 15(7): 841-852.

Morin D. Introduction to Classical Mechanics. Cambridge University Press, New York 2008.

Moser MB and Moser EI. Functional differentiation in the hippocampus. Hippocampus 1998; 8(6): 608-19.

Munoz-Lopez MM, Mohedano-Moriano A and Insausti R. Anatomical pathways for auditory memory in primates. Frontiers in Neuroanatomy 2010; 4(129). doi: 10.3389/fnana.2010.00129

Nelson SM, McDermott KB, Wig GS, Schlagger BL and Petersen SE. The critical roles of localization and physiology for understanding parietal contributions to memory retrieval. Neuroscientist 2013; 19: 578-591.

O'Keefe DJ. Place units in the hippocampus of the freely moving rat. Experimental Neurology 1976; 51(1): 78-109.

O'Keefe J and Dostrovsky J. The hippocampus as a spatial map. Preliminary evidence from unit activity in the freelymoving rat. Brain Research 1971; 34(1): 171-175.

O'Keefe J and Nadel L. The Hippocampus as a Cognitive Map. Oxford University Press, 1978.

Penrose R. The Road to Reality. Vintage Books, London 2005.

Petkov V. Minkowski Spacetime: A Hundred Years Later. Springer, Berlin 2010

Popper AN. Music Perception. Springer 2010; 150.

Priest SH. Encyclopedia of Science and Technology Communication. SAGE Publications 2010.

Rolls ET. The mechanisms for pattern completion and pattern separation in the hippocampus. Frontiers in Systems Neuroscience 2013; 7(74). doi: $10.3389 /$ fnsys.2013.00074

Roberts $\mathrm{T}$ and Schleif S. What is the experimental basis of special relativity? Usenet Physics FAQ 2007. 
Schacter DL, Gilbert DT and Wegner DM. Semantic and episodic memory. Psychology, 2nd Ed., 240-241. Worth Incorporated, New York, 2011.

Serino S and Riva G. What is the role of spatial processing in the decline of episodic memory in Alzheimer's disease? The "mental frame syncing" hypothesis. Frontiers of Aging Neuroscience 2014; 6(33). doi: $10.3389 /$ fnagi.2014.00033

Sieb RA. The emergence of consciousness. Medical Hypotheses 2004; 63(5): 900-904.

Sieb RA. The emergence of emotion. Activitas Nervosa Superior 2013; 55(4): 115-145.

Sieb RA. Memory in four dimensions. In: M.Sakakibara \& I.Etsuro (Eds.), Memory Consolidation, Chapter 13, 259313. Nova Science Publishers, Inc., New York, 2015.

Sieb RA. Four-Dimensional Consciousness. relativisticconsciousness.com 2016.

Steinvorth S, Levine B and Corkin S. Medial temporal lobe structures are needed to re-experience remote autobiographical memories: evidence from H.M. and W.R.. Neuropsychologia 2005; 43: 479-496.

Sumner B. The effect of experience on the perception and representation of dialect variants. Journal of Memory and Language. Elsevier Inc. 2009.

Tipler P and Llewellyn R. Modern Physics (5th ed.). W.E. Freeman Company 2008; 160-161.
Tulving E. Elements of Episodic Memory. Oxford University Press, New York, 1983.

Ullman MT. Contributions of memory circuits to language: The declarative/procedural model. Cognition 2004; 92: 231270.

Uncapher MR and Wagner AD. Posterior parietal cortex and episodic encoding. Insights from fMRI subsequent memory effects and dual-attention theory. Neurobiology of Learning \& Memory 2005; 91: 139-154.

Wagner AD, Shannon BJ, Hahn I and Buckner RL. Parietal lobe contributions to episodic memory retrieval. Trends in Cognitive Science 2005; 9: 445-453.

Weinberg S. Dreams of a Final Theory: The Scientists Search for the Ultimate Laws of Nature. Knopt Doubleday Publishing Group 1993.

Weiten W. Psychology: Themes and Variations, 8th ed., Wadsworth/Cengage Learning 2010.

Wendelken C. Meta-analysis: how does posterior parietal cortex contribute to reasoning? Frontiers of Human Neuroscience 2015; 8(1042). doi: 10.3389/fnhum.2014.01042

Wolfe JM, Kluender KR, Levi DM, Bartochuk LM, Herz RS, Klatsky RL and Lederman SJ. Gestalt grouping principles. Sensation and Perception (2nd ed.). Sinauer Associates 2008; 78-80. 